\title{
Holes in Spacetime: Some Neglected Essentials
}

\author{
Trevor Teitel \\ New York University \\ April 23, 2019 \\ Forthcoming in The Journal of Philosophy
}

\begin{abstract}
The hole argument purports to show that all spacetime theories of a certain form are indeterministic, including the General Theory of Relativity. The argument has given rise to an industry of searching for a metaphysics of spacetime that delivers the right modal implications to rescue determinism. In this paper, I first argue that certain prominent extant replies to the hole argument-namely, those that appeal to an essentialist doctrine about spacetime-fail to deliver the requisite modal implications. As part of my argument, I show that threats to determinism of the sort brought out by the hole argument are more general than has heretofore been recognized. I then use these results to propose a novel essentialist doctrine about spacetime that successfully rescues determinism, what I call sufficiency metric essentialism. However, I go on to argue that once we realize what an essentialist doctrine about spacetime must look like in order to address the hole argument, we should reject all such doctrines, because they can't fulfill their ambition of improving on standard modal replies to the argument. I close by suggesting some lessons for future work on spacetime and the metaphysics of physics more broadly, and also drawing some general morals for contemporary metaphysics, in particular about (i) whether essence can be used to articulate a precise structuralist doctrine, and (ii) the relationship between essence and modality.
\end{abstract}

Call a proposition qualitative just in case it's not about any particular objects; call all other propositions haecceitistic (I'll also call these non-qualitative propositions). For example, the proposition that someone is sitting is qualitative, whereas the proposition that Larry is sitting is haecceitistic. Call a property $F$ qualitative just in case the proposition that something has $F$ is qualitative; call all other properties haecceitistic or non-qualitative. For example, the property of liking someone is qualitative, whereas the property of liking Larry is haecceitistic. With these distinctions in hand, we can state two senses in which some laws of nature $L$ may be deterministic:

Full Determinism: For all metaphysical possibilities $w$ and $w^{\prime}$ where $L$ is true, if there is a time $t$ at both $w$ and $w^{\prime}$ such that $t$ has the same intrinsic properties at both $w$ and $w^{\prime}$, then $w$ and $w^{\prime}$ agree on the truth value of every proposition.

Qualitative Determinism: For all metaphysical possibilities $w$ and $w^{\prime}$ where $L$ is true, if there is a time $t$ at $w$ and a time $t^{\prime}$ at $w^{\prime}$ such that $t$ has the same 
qualitative intrinsic properties at $w$ that $t^{\prime}$ has at $w^{\prime}$, then $w$ and $w^{\prime}$ agree on the truth value of every qualitative proposition. ${ }^{1}$

Ever since Earman and Norton (1987) resurrected Einstein's hole argument, and set it loose in the foundational discussion of spacetime theories, an industry has grown up around searching for doctrines which ensure that the qualitative determinism of the laws of Einstein's General Theory of Relativity (hereafter 'GR') implies that those laws are also fully deterministic. $^{2} \mathrm{I}^{\prime} \mathrm{m}$ ultimately going to use this challenge to argue for certain doctrines in the metaphysics of spacetime and the metaphysics of modality, but before getting there let's first see what all the fuss is about.

\section{The Hole Story}

Some quick preliminaries before stating Earman and Norton's argument. First, I'll take it for granted that metaphysical modality obeys the modal logic S5. Second, I'll assume throughout that the nomic possibilities at any metaphysical possibility $w$ just are the metaphysical possibilities where the laws of nature at $w$ are true. This assumption is neutral between the standard view that the space of nomic possibilities is properly included in the space of metaphysical possibilities, and its main alternative where these two spaces are identical. I'll also assume that only qualitative propositions can be laws of nature, and hence that the haecceitistic modal constraints needed to get from qualitative to full determinism must stem from some constraints on metaphysical modality. Finally, I'll write throughout as if the actual laws of nature are those of GR. So my use of 'nomic possibilities' without qualification means metaphysical possibilities where the laws of GR are true.

Earman and Norton pitch their hole argument as stemming from GR's diffeomorphism invariance, which they take to imply that, for every nomic possibility $w$, there's a distinct nomic possibility for every way of smoothly permuting some spacetime points at $w$ but

\footnotetext{
${ }^{1} \mathrm{~A}$ few comments about these definitions. First, arguably the definitions should be phrased in terms of arbitrarily small temporal intervals rather than particular times (so as to allow rates of change into the initial data when evaluating whether some laws are deterministic), but I'll ignore this subtlety throughout. Second, the intrinsic qualifiers are intended to exclude properties that directly encode information about different times as being amongst those we must consider when checking whether two metaphysical possibilities agree at a time. For instance, consider the property of being a time fifty years before a time at which there is a nuclear war. Supposing some time $t$ has this property, it's not one of $t$ 's intrinsic properties. Without some ideology to rule out such properties, any laws whatsoever will trivially turn out deterministic on either definition. Note the qualifiers are intended to allow properties concerning the material objects (if any) located at some time to nonetheless count as intrinsic properties of that time. For discussion of distinctions between different varieties of determinism akin to my qualitative vs. full determinism see Belot (1995), Brighouse (1997), Melia (1999) and Hawthorne (2006). Some of these works discuss notions of determinism that go beyond qualitative determinism but fall short of full determinism. These intermediate options don't affect the central dialectic in what follows, however, so I'll set them aside.

${ }^{2}$ See Pooley (2013, Section 7) and Norton (2015) for recent overviews of the hole argument and the menu of possible replies. As Earman and Norton point out $(1987,517-518)$, their argument in fact applies to a general class of classical (non-quantum) field theories, sometimes called "local spacetime theories", not just to GR, but in the main text I'll focus on GR (our current best spacetime theory).
} 
leaving everything qualitatively unchanged. ${ }^{3}$ Earman and Norton then invite us to consider two such nomic possibilities, yet where the second is generated from the first by smoothly permuting the spacetime points only within some subregion of spacetime (the "hole") that lies to the future of some time (Cauchy surface). ${ }^{4}$ They then point out that such possibilities are a counterexample to GR's full determinism: the possibilities agree on all propositions up to some time, but then go on to disagree over the non-qualitative question of which of some fixed class of spacetime points (those inside the hole) play which qualitative roles. Earman and Norton took spacetime substantivalism to be the crucial doctrine that forces commitment to such merely haecceitistically different possibilities, and also took this armchair demonstration that GR isn't fully deterministic to be problematic, and so pitched their hole argument as supporting some sort of relationism about spacetime in the context of GR. Given the familiar difficulties that plague attempts to develop an adequate relationist view (see Earman (1989)), instead the predominant reaction has been to seek a substantivalist metaphysics of spacetime that addresses the argument.

One immediate reaction you might have is simply to grant the argument, yet contend that substantivalists needn't worry about the merely non-qualitative failures of full determinism that the argument brings out. Indeed some argue that any reasons to want our theories to be deterministic support only qualitative determinism (e.g., Brighouse (1997)), and all parties in the debate grant GR's qualitative determinism.

Earman and Norton's response here is that even if you don't regard full determinism as an important feature for physical theories to have, you should nonetheless be concerned that the hole argument reveals failures of full determinism to be relatively trivial to conjure up; in Earman and Norton's own words, "a metaphysics which automatically decides in favour of indeterminism is [...] unacceptable. Determinism may fail, but if it fails it should fail for a reason of physics" $(1987,524)$ (see also Earman $(1989,180-181)$ ). There's some intuition here, but I've never been that moved by this reply. Whether some laws are fully deterministic depends on which objects there are, and what's metaphysically possible for these objects. These aren't the kinds of questions we should expect to read directly off of our best physics. Rather, they turn at least in part on issues in metaphysics, and hence apriori reflection could in principle incline us towards deeming some laws not fully deterministic. $^{5}$

\footnotetext{
${ }^{3}$ In fact diffeomorphism invariance is a purely mathematical constraint on the solution space of a general relativistic theory, which on its own has no consequences for nomic modality. I further discuss this claim, as well as its implications for theorizing about spacetime theories in my (2019a). Throughout I heed the morals of that paper by discussing the hole argument directly in modal terms, rather than instead focussing on solution spaces and their representational capacities. We'll see some fruits of this methodological shift throughout.

${ }^{4}$ I'll follow standard practice in the literature and focus on the globally hyperbolic sector of GR (i.e. focus on spacetimes that admit a Cauchy surface), and assume that at possibilities where GR is true all and only Cauchy surfaces are times. However, we'll see in section 6 that relaxing these restrictions arguably strengthens the case for my conclusions.

${ }^{5}$ Indeed Hawthorne (2006) argues against full determinism (his "de re determinism") on purely apriori grounds having nothing to do with physics. His arguments turn on haecceitistic propositions about macroscopic material objects, which I won't discuss here.
} 
So should substantivalists just call the argument sound and move on, on the grounds that qualitative determinism is the only sense of determinism that matters, and hence the purely haecceitistic failures of full determinism that the argument brings out aren't objectionable? I don't think so: anyone sympathetic to a certain popular position in the metaphysics of laws should arguably be invested in rescuing GR's full determinism. The position I have in mind is any non-Humean governing view, which regards our laws of nature as explaining in some metaphysically robust sense why our universe evolves in the way it does (e.g. the primitivist view defended by Maudlin (2007)). Proponents of such views, perhaps motivated in part by something like the PSR, should be reluctant to concede that our laws of nature are simply silent on a huge range of non-qualitative facts. It's hard to move past picture-thinking here without digressing into different ways of cashing out the governing metaphor. Still, I don't anticipate that any of the candidates for doing so will dispel the strong prima facie tension between conceding failures of GR's full determinism and regarding our laws as determining why our universe evolves in the way it does. Henceforth I'm going to take for granted that we have some reason to be invested in rescuing GR's full determinism, which is a presupposition of the vast majority of work on the hole argument.

Having put aside the concessive reply, the challenge is to find some doctrine in the metaphysics of modality or spacetime to ensure that GR's qualitative determinism implies its full determinism. Much ink has been spilled propounding and evaluating replies that take on this challenge (see footnote 2 for some surveys). I'm wading into these murky waters here because in my view one prominent sort of such a reply-that appeals to a notion of essence combined with some claim about what's essential to a spacetime point or region (the most central example of which is Maudlin's (1988; 1990) metric essentialism) — has yet to be adequately worked out. However, we'll see in the next section that it's easy to get into a frame of mind where some such essentialist reply must be correct. I'll ultimately argue that getting clear on what an essentialist doctrine about spacetime must look like in order to address the hole argument suggests that no reply to the argument that takes even the general form of an essentialist reply can succeed. This result will cause us to reconsider the motivations for such a reply, which will have important implications for contemporary metaphysics as well as future work on spacetime.

Here's the plan. I'll first argue that natural formulations of the essentialist reply, including all extant formulations, offer doctrines that simply fail to address the issues brought out by the hole argument (i.e. the doctrines don't ensure that GR's qualitative determinism implies its full determinism). As part of my argument, I'll show that threats to determinism of the sort brought out by the hole argument are more general than has heretofore been recognized. I'll then use these results to propose a novel essentialist doctrine about spacetime that successfully bridges GR's qualitative and full determinism, what I'll call sufficiency metric essentialism. However, I'll go on to argue that once we realize what an essentialist 
doctrine about spacetime must look like in order to address the hole argument, we have reason to reject all such doctrines, because they can't fulfill their ambition of improving on standard modal replies to the argument (which I'll outline in a moment). I'll then close by suggesting some lessons for future work on spacetime and the metaphysics of physics more broadly, and also drawing some general morals for contemporary metaphysics, in particular about (i) whether essence can be used to articulate a precise structuralist doctrine, and (ii) the relationship between essence and modality. I should also flag that the conclusions I'll reach about the correct form and relative merits of the essentialist reply carry over to several other analogous debates where local gauge symmetries pose a threat to full determinism (e.g. those that arise in foundational discussions of electromagnetism), but in what follows I'll discuss only GR and the hole argument. Moreover, as I'll explain in the conclusion, although I focus on essentialist replies, the issues I'll raise for such replies arise for any attempt to address the hole argument by appealing to some hyperintensional resources while still countenancing objects (in particular, spacetime points).

Before we get going, in the next section I'll sketch the main replies to the argument and some worries for them. Doing so will convey why anyone not inclined to just grant the failure of full determinism from the get-go should be invested in seeking an essentialist doctrine about spacetime that can address the hole argument (bridge GR's qualitative and full determinism). Again, I'll ultimately argue that no such view can succeed, so we'll have cause to revisit some of these worries later on.

\section{Motivating Essentialist Doctrines About Spacetime}

Let's first get some of the modal doctrines relevant to the hole argument on the table:

Anti-Haecceitism: No metaphysical possibilities differ without differing qualitatively.

GR-Anti-Haecceitism: No metaphysical possibilities where the laws of GR are true differ without differing qualitatively.

SP-Anti-Haecceitism: No metaphysical possibilities differ either over which spacetime points there are, or over which spacetime points instantiate which spatiotemporal properties and relations, without also differing over the qualitative structure of spacetime.

It's straightforward to see that GR-anti-haecceitism (and hence anti-haecceitism itself, which is strictly stronger) ensures that GR's qualitative determinism implies its full determinism. Both doctrines rule out there being metaphysical possibilities where the laws of GR are true that differ merely haecceitistically. As a result, both doctrines ensure that there can't be purely haecceitistic failures of GR's full determinism, and hence that GR can fail to be fully deterministic only if it fails to be qualitatively deterministic as well, which is exactly 
the result those responding to the hole argument were after. Because we're focussing on GR, I'll discuss GR-anti-haecceitism instead of anti-haecceitism itself. Doing so allows me to stay neutral on whether there are independent reasons to reject anti-haecceitism (e.g. stemming from metaphysical possibilities with classical spacetimes that differ merely over uniform spatial translations). I'll also assume that SP-anti-haecceitism implies GR-antihaecceitism, which follows given the assumption that metaphysical possibilities where the laws of GR are true can differ merely haecceitistically only if they differ either over which spacetime points there are or over which spacetime points instantiate which spatiotemporal properties and relations. This latter assumption is common throughout the literature on the hole argument (albeit often only implicit) - where the concern is to address only spacetime-centric haecceitistic threats to full determinism-and explains the focus on metaphysical doctrines that purport to secure SP-anti-haecceitism. ${ }^{6}$

Many replies to the argument end up securing at least one of these three varieties of anti-haecceitism, but they differ over whether they try to further explain the modal doctrine in independent terms, and if so how. Perhaps the most popular reply to the argument in the literature is to simply supplement substantivalism with at least one of these varieties of anti-haecceitism, and remain silent about whether there's any further explanation of why this constraint on metaphysical modality obtains. ${ }^{7}$ We just saw that any of those three anti-haecceitist doctrines suffices to bridge the gap between GR's qualitative and full determinism, and hence address the hole argument.

However, many will want to go further than simply laying down some variety of antihaecceitism and calling it a day: such a strong constraint on metaphysical modality seems mysterious, and the sort of thing that cries out for further explanation. ${ }^{8}$ Indeed, a prominent trend in recent metaphysics is a suspicion of theorizing directly in terms of modality, and a shift towards formulating theses in allegedly hyperintensional terms and trying to explain modal claims in terms of these; as Schaffer puts it, "modal correlation is at best

\footnotetext{
${ }^{6}$ The assumption brackets the tricky issue of whether GR vindicates a supersubstantivalist metaphysics; in particular, the assumption takes for granted that there aren't objects other than spacetime points (such as material objects like particles) at any metaphysical possibilities where the laws of GR are true.

${ }^{7}$ For some examples see Brighouse (1994), Hoefer (1996) and Pooley (2006). Field (1984) adopts antihaecceitism when responding to classical Leibniz shift arguments, and so would likely use the doctrine to address the hole argument as well. Note that what often gets called "sophisticated substantivalism" is the combination of spacetime substantivalism with any view that implies anti-haecceitism, irrespective of whether the modal constraint is explained in independent terms.

${ }^{8}$ Here's one way to drive this home. (I'll direct the worry at SP-anti-haecceitism, but an analogous worry applies to the other anti-haecceitist doctrines.) SP-anti-haecceitism by itself-in particular, the doctrine without the addition of some extremely strong necessary modal condition on what spacetime points must be like if they're to be spacetime points-is committed to seemingly brute facts about which particular qualitative roles spacetime points can play across metaphysical possibilities. This is true because SP-anti-haecceitism implies that each complete qualitative geometric structure can have only one possible non-qualitative realization, yet the lack of an extremely strong necessary modal condition ensures that it's not the case that any distinct complete qualitative geometric structures are realized by disjoint classes of spacetime points. We'll see in section 6 that accepting some extremely strong necessary modal condition on spacetime points is independently problematic. So those moved by this worry from avoiding seemingly brute facts should reject SP-anti-haecceitism, which leaves open a picture where any spacetime points could've played any qualitative roles.
} 
a symptom" $(2009,364)$. One stark instance of this trend is the shift away from supervenience and towards grounding as the relevant ideology for stating doctrines like physicalism, though numerous other examples of the trend could be given. ${ }^{9}$ The trend also goes hand in hand with rejecting the doctrine that modal facts are fundamental, and instead claiming that modal facts obtain in virtue of some more fundamental facts (e.g. facts about essence). Perhaps the thought here is that grounding modal facts in other terms leaves us with fewer mysterious facts (however we'll see reason to doubt this inference in the conclusion). Anyone sympathetic to this recent revolt against modality has reason to try to further explain the modal constraints needed to bridge the gap between GR's qualitative and full determinism. Moreover, essentialist replies-which purport to explain the requisite modal constraints in terms of essentialist ones-are the most promising option to try here: we'll soon see that the most popular and well worked-out attempt to explain modal theses in hyperintensional terms appeals to essence, following Fine (1994). So the desire to further explain the modal constraints from the hole argument provides ample motivation to adopt an essentialist reply.

The main alternative to an essentialist reply for those hoping to further explain the requisite modal constraints is some form of "structuralism" about spacetime, defended by "ontic structural realists". What does this doctrine say? Good question: it's hard to find a doctrine about spacetime that hasn't at some point been championed under the banner of structuralism. Even essentialist replies can be found amidst the numerous things structuralists say when expounding their views, and at times structuralism just amounts to some variety of anti-haecceitism. ${ }^{10}$ To my mind, the best option currently on offer for an intelligible and distinctive structuralist view is a holistic and purely qualitative extension of the traditional bundle theory (sketched by Van Cleve (1985) and defended at most length by Dasgupta $(2009 ; 2011 ; 2017)$ under the heading of "algebraic generalism"). Roughly, on this view the fundamental description of the world describes the pattern of which qualitative fundamental properties and relations are compresent with which others. However, this view is still very much under development. First off, how to even understand talk of objects and haecceitistic facts in this setting is an open question (see Russell (2016) for

\footnotetext{
${ }^{9}$ For some explicit expressions of suspicion about the usefulness of theorizing directly in terms of modality see Arntzenius (2012, 1-2) and Sider (2011, ch.12). For discussion of this trend in the context of the hole argument see Dasgupta (2011), who uses it to motivate trying to further explain SP-anti-haecceitism via a qualitativist doctrine, sketched below in the main text. For notable push-back against this shift away from theorizing directly in terms of modality see Williamson $(2013 ; 2016)$.

${ }^{10}$ The literature on ontic structural realism is vast. See Ladyman (2014) for a general overview and Ladyman \& Ross (2007) for a book-length defense. For skepticism about whether there's a precise and intelligible view in the vicinity that doesn't collapse into some familiar alternative see Pooley (2006), Dorr (2010), and Greaves (2011). (These works will also give you a sense of the numerous inequivalent views that often get lumped together in discussions of structuralism.) Schaffer $(2010 \mathrm{a}, 365)$ explicitly proposes an essentialist thesis under the heading of structuralism though there he's neutral on whether essence can be understood in terms of modality; see also Lowe $(2012,231)$. An essentialist thesis is also one clear doctrine to extract from the "moderate ontic structural realism" of Lam and Esfeld (indeed they sometimes explicitly state their doctrine in terms of essence, see for example their $(2012,247))$.
} 
a noble recent attempt to explore some options here). Yet without such an account we can't even begin to assess whether the view implies some variety of anti-haecceitism, or more generally bridges the gap between GR's qualitative and full determinism (because to do so we need to know the truth conditions in this object-free setting of putatively nonqualitative propositions which purport to be about particular objects). Second, there's a case to be made that the view hasn't in fact freed itself of a commitment to objects (see Turner (2017)). Neither of these worries poses a decisive objection to the view, but they show that it remains to be seen whether there's a coherent and defensible vision of spacetime in the vicinity of structuralist slogans that's distinct from the other options.

Later on I'll suggest that the essentialist metaphysics of spacetime I arrive at should be of interest to structuralists searching for a precise conception of spacetime that vindicates some of their slogans. Though, as mentioned above, I'll ultimately argue that the view doesn't improve on the familiar modal replies that simply lay down some variety of antihaecceitism and say nothing else. Indeed, I'll suggest in the conclusion that reflecting on what an essentialist doctrine must look like in order to address the hole argument gives us reason to reconsider whether we must further explain the relevant modal constraints, and even to reconsider aspects of the recent revolt against modality in contemporary metaphysics.

\section{Metric Essentialism}

Let's now see exactly what an essentialist doctrine that purports to address the hole argument might look like. The most prominent such reply is Maudlin's metric essentialism, which is standardly stated as the doctrine that a spacetime point's metric relations are essential to it. It's easy to see why one might think that such a view could help with the hole argument. Given some nomic possibility, the view predicts that we can't generate another nomic possibility by freely permuting which spacetime points instantiate which properties and relations: any such permutation requires changing some points' spatiotemporal properties and relations, which would conflict with the points' essential properties.

Unfortunately, metric essentialism hasn't been made more precise than the informal statement just given. As a result, we're not yet able to even ask whether the view vindicates the precise modal constraint stemming from the hole argument (bridging GR's qualitative and full determinism). In this section I'll try to make the metric essentialist's vision more precise, and show that there are two importantly different essentialist doctrines one might take metric essentialism to amount to.

\subsection{Some Essential Preliminaries}

We first need to spell out three preliminaries about the notion of essence and how it relates to metaphysical modality. 
First, there's a long tradition of reducing essence to modality, for instance claiming that a property is essential to some object or plurality of objects in virtue of it being metaphysically necessary that the object or plurality of objects has the property. (I'll use 'reduces to', 'holds in virtue of', and 'is grounded in' interchangeably throughout.) This view is widely held to have been refuted by Fine's (1994) influential counterexamples. The most famous one involves Socrates and his singleton set. Given standard assumptions in modal set theory, necessarily if Socrates exists then he's contained in his singleton. Yet many have a strong judgment that, assuming both Socrates and his singleton exist, it's essential to singleton-Socrates to contain Socrates yet not essential to Socrates to be contained in his singleton, and moreover that only the former essentialist truth explains why necessarily if Socrates exists then he's contained in his singleton. Taking these essentialist judgments at face-value gives a straightforward counterexample to the standard modal account of essence. For the purposes of developing the best version of the essentialist response, I'll take on the now standard view that the notion of essence isn't to be cashed out in modal terms, and that we nevertheless have sufficient pre-theoretical handle on the notion to render our discussion tractable, despite viewing these as open issues (see my (2019b) for some reservations).

Second, we're going to want to link essence to modality, and the arguments of modal operators are sentences not predicates. So throughout we'll adopt the now standard practice of saying that propositions rather than properties are essential to objects. It's not hard to translate the more traditional way of regimenting essence into these terms: that some property $F$ is essential to some object $o$ can be interpreted as the claim that the proposition that $o$ is $F$ is essential to $o$.

Finally, the metric essentialist will need some link between essence and metaphysical modality, because she wants to use her essentialist doctrine to further explain why GR's qualitative determinism implies its full determinism. One option here would be to maintain that neither essence nor metaphysical modality is reducible to the other, yet hold that it's just a brute correlation that necessarily all propositions essential to some object or plurality of objects are also metaphysically necessary. A second option would be to explain why essence has implications for metaphysical modality via an underlying reductive doctrine, which reverses the standard order of priority by reducing metaphysical modality to essence. The canonical reduction of this sort is due to Fine (1994), which says that a proposition $p$ is metaphysically necessary in virtue of there being some object or plurality of objects such that $p$ is essential to that object or plurality of objects. ${ }^{11}$ I suspect that all proponents of metric essentialism will go for this second option-where they explain the relevance of their essentialist thesis to modal matters via Fine's reduction of modality to

\footnotetext{
${ }^{11}$ This formulation of the reduction is equivalent to Fine's own (a proposition is metaphysically necessary in virtue of being essential to the plurality of all objects whatsoever) given Fine's background theory of essence (in particular his monotonicity assumption). The formulation in the main text is more effective at giving a clear picture of how the reduction to essence works, so I'll stick with it throughout.
} 
essence. The first option is in tension with the kinds of considerations we saw above that would motivate proponents of essentialist replies to the hole argument to go beyond some variety of anti-haecceitism in the first place.

Now I'm well aware that the very mention of the notion of essence will have many philosophers of physics running for the hills, so I should emphasize that readers who reject the intelligibility of a notion of essence that's not reducible to metaphysical modality will still find much of interest in what follows, and may be sympathetic to the conclusions I ultimately reach about the merits of essentialist replies. Indeed such readers can take on many of the morals I'll draw by extracting the modal content of the various essentialist theses I'll consider (using Fine's reduction). For example, I'll argue that neither variety of metric essentialism we'll see in this section has the right modal implications to address the hole argument, and so my arguments apply equally well to those who regard essentialist theses as just colourful ways to express corresponding doctrines about metaphysical modality. ${ }^{12}$

\subsection{Qualitative and Non-Qualitative Metric Essentialism}

Let's turn to the two essentialist doctrines you might read into the metric essentialist's slogan that spacetime points stand in their metric relations essentially. One will be a purely qualitative version of the doctrine, whereas the other will build haecceitistic facts into a spacetime point's essence.

Call a proposition the complete spacetime description of some metaphysical possibility $w$ iff it details the complete pattern of instantiation at $w$ of the fundamental spatiotemporal properties and relations, and nothing else. ${ }^{13}$ Note these will be non-qualitative propositions, because they detail which particular spacetime points instantiate which fundamental spatiotemporal properties and relations; we'll see an example shortly. Given a complete spacetime description of some metaphysical possibility $w$, we can define, for any spacetime point $o$ at $w$, the complete spacetime description of $w$ according to $o$ : the proposition that if $o$ is a spacetime point then the complete spacetime description of $w$ obtains.

For any metaphysical possibility $w$, and any spacetime point $o$ at $w$, we now define $w$ 's complete qualitative spacetime description according to $o$ : the proposition that if $o$ is a spacetime point then the complete qualitative pattern of instantiation at $w$ of the fundamental spatiotemporal properties and relations obtains and $o$ plays the qualitative geometric role in that structure that it in fact plays at $w$. Given a sentence expressing the complete spacetime description of some possibility according some spacetime point $o$, if we existentially

\footnotetext{
${ }^{12}$ Indeed Maudlin's $(1988 ; 1990)$ discussions of metric essentialism—which appeared before Fine's influential arguments against modal analyses of essence-are neutral on whether essentialist theses are just shorthand for doctrines concerning metaphysical modality. Again, most of the points I'll make apply either way.

${ }^{13} \mathrm{I}^{\prime} \mathrm{ll}$ write as if it's non-contingent which properties and relations are fundamental, though of course contingent which fundamental properties and relations are instantiated. Doing so simplifies the exposition here, but nothing I say is wedded to this view.
} 
generalize on every occurrence of a name for a spacetime point besides the name for $o$, the resulting quantified sentence expresses the complete qualitative spacetime description of that possibility according to $o$.

To give a toy example, suppose $w$ contains three spacetime points $a, b$, and $c$. Let $R$ be a two-place 'is adjacent to' relation. Suppose the fundamental spatiotemporal facts at $w$ are $R a b, R b a, R b c, R c b, \neg R a c, \neg R c a$. Then the complete spacetime description of $w$ according to $a$ is the proposition that if $a$ is a spacetime point then $R a b \wedge R b a \wedge R b c \wedge R c b \wedge \neg R a c \wedge$ $\neg R c a \wedge$ there are no spacetime points other than $a, b$, and $c$. And the complete qualitative spacetime description of $w$ according to $a$ is the proposition that if $a$ is a spacetime point then $\exists x \exists y(R a x \wedge R x a \wedge R x y \wedge R y x \wedge \neg R a y \wedge \neg R y a \wedge$ there are no spacetime points other than $a, x$, and $y)$.

With these definitions in hand, we can state our two varieties of metric essentialism:

Non-Qualitative Metric Essentialism: For every metaphysical possibility $w$, and every spacetime point $o$ at $w$, the complete spacetime description of $w$ according to $o$ is essential to $o$.

Qualitative Metric Essentialism: For every metaphysical possibility $w$, and every spacetime point $o$ at $w$, the complete qualitative spacetime description of $w$ according to $o$ is essential to $o$.

Here are two precise doctrines one might have taken metric essentialism to amount to. I'll now argue that it's simply a mistake to take metric essentialism to address the hole argument: both the qualitative and non-qualitative understandings of the doctrine fail to deliver the right modal implications to bridge the gap between GR's qualitative determinism and its full determinism. Although both doctrines suffice to address Earman and Norton's original example, we'll see that the relevant threats to GR's full determinism are more general than has heretofore been recognized. In the next section, I'll argue that proponents of either form of metric essentialism must accept some nomic contingency in which spacetime points there are, which will open the door to my argument in the section after next that such contingency spells trouble for qualitative metric essentialism. I'll then turn to the problems with non-qualitative metric essentialism. These results will point the way towards what an essentialist doctrine about spacetime that in fact addresses the hole argument would have to look like, which we'll then see to nevertheless be problematic.

\section{The Spinozist Threat}

In this section, I'll argue that metric essentialists of either sort must reject the following doctrine:

SP-Necessitism: All metaphysical possibilities where the laws of GR are true agree about which spacetime points there are. 
We can see why by revisiting a standard objection to metric essentialism in the setting of GR, stemming from counterfactuals like 'if the sun had been more massive then the region of spacetime the sun actually occupies would have had higher curvature' ${ }^{14}$ Counterfactuals of this sort seem like they should turn out true given GR (because of the Einstein equation correlating the stress-energy-momentum of matter with the curvature of spacetime). But, the objection runs, such counterfactuals turn out false if we embrace metric essentialism. (Recall from section 1 that I'm writing throughout as if the actual laws of nature are those of GR to ease exposition.) To see the alleged reason for this, let ' $R$ ' name the region of spacetime occupied by the sun (where I'll assume that regions are mereological fusions of spacetime points, and so can't change their parts). Then, given either form of metric essentialism, $R$ itself couldn't have had higher curvature than it actually does: $R$ could have higher curvature only if the metric relations of the points that compose $R$ were to be different, but this isn't possible given either qualitative or non-qualitative metric essentialism. So because the truth of the problematic counterfactual requires that if the sun had been more massive then the region it actually occupies would have had higher curvature, the metric essentialist must concede that the counterfactual and others like it are false, because such counterfactuals conflict with her essentialist doctrine. ${ }^{15}$

We can state an analogous problem directly in terms of nomic modality, which lets us side-step delicate issues about the semantics of counterfactuals. The reasoning above straightforwardly generalizes to show that if we assume SP-necessitism and either qualitative or non-qualitative metric essentialism then every true proposition detailing the pattern of instantiation of metric relations is nomically necessary (where, as mentioned in section 1, my unqualified uses of 'nomically necessary' express truth at every metaphysical possibility where the laws of GR are true). As a result, given the Einstein equation, every true proposition whose truth value can't change without varying the value of the stress-energymomentum tensor at some point in spacetime will turn out nomically necessary. This isn't quite the Spinozist result that all true propositions are nomically necessary, but it's close: most non-trivial true propositions about the physical world will turn out nomically necessary. For instance, the result implies that, holding fixed the laws of nature, the sun couldn't have been more massive than it actually is, I couldn't have blinked one millisecond before I actually blinked, and so on.

Once metric essentialists of either sort reject SP-necessitism, and hence allow that which

\footnotetext{
${ }^{14}$ For discussion see Maudlin $(1988,88)$ and $(1990,550-551)$, and also Earman $(1989,201-202)$ and Brighouse (1994, 119-120).

${ }^{15}$ Here's a bit more detail on why this is true. The Einstein equation of GR correlates the stress-energymomentum tensor (defined from whatever matter fields there are) with the Einstein tensor. This latter tensor is defined from the Riemann curvature tensor and the metric. In principle you could have a manifold with a connection besides the Levi-Civita connection (the unique metric-compatible and torsion-free connection definable from the metric). However, it's standard to restrict attention to models of GR that don't contain a redundant non-metric-compatible connection, in which case the Riemann tensor itself is definable from the metric, and hence the metric alone fixes the Einstein tensor. The result is that changes to the stress-energymomentum tensor, and hence the Einstein tensor, will always engender changes to the metric.
} 
spacetime points there are varies across nomic possibilities, they have the resources to answer these objections. True, both varieties of metric essentialism will still rule out the counterfactual above that imposes a constraint on $R$ (the region the sun actually occupies). However, either doctrine minus SP-necessitism can vindicate the truth of nearby counterfactuals like 'if the sun had been more massive then it would have occupied a region with higher curvature than the region it actually occupies'. Counterfactuals of this sort impose only a qualitative constraint on what spacetime must be like if the sun had been more massive, and so can be made true by nomic possibilities where the sun occupies a region composed of points that are distinct from the points that compose $R$ (and hence some region other than $R$ ). Metric essentialists of either sort can then say that our judgments about which counterfactuals must turn out true given the truth of GR are sufficiently vindicated by counterfactuals which impose only qualitative constraints on how the metric relations of spacetime must change in response to changes in matter. A similar reply dispels the threat of Spinozism: I could have blinked one millisecond before I actually blinked without the laws of GR becoming false, just if I were to have done so I would have occupied a numerically distinct region from the one I actually occupy (because this region must have a different value of the stress-energy-momentum tensor and hence instantiate different metric relations). We see, then, that provided metric essentialists reject SP-necessitism, they can vindicate enough of our pre-theoretical modal judgments about spacetime to allay worries stemming from this front. ${ }^{16}$

\section{A Revised Hole Argument}

We just saw that metric essentialists of either sort must reject SP-necessitism, and so embrace some nomic contingency in which spacetime points there are. I'll now argue that allowing for such nomic contingency poses a problem for qualitative metric essentialism. I'll turn to the problems with non-qualitative metric essentialism in the next section.

Recall that Earman and Norton's hole argument brings out a failure of GR's full determinism using qualitatively identical nomic possibilities that agree about which spacetime points there are, but differ over which of those points play which qualitative geometric roles after some time. Qualitative metric essentialism blocks this argument by tying points to their qualitative geometric roles via an essentialist constraint, which combined with the reduction of modality to essence we saw above ensures that there can't be distinct nomic

\footnotetext{
${ }^{16}$ I should flag that SP-necessitism is independent of Williamson's (2013) doctrine of necessitism itself-the doctrine that necessarily everything is necessarily something, or more colloquially that necessarily everything necessarily exists. So metric essentialists can reject SP-necessitism yet nevertheless embrace necessitism should they be so inclined. This is relevant because I've argued in my (2019b) that Fine's reduction of modality to essence can succeed only if it's paired with necessitism (given also the assumption above that metaphysical modality obeys the modal logic S5). Thus insofar as metric essentialists must countenance Fine's reduction (as I suggested above), they must arguably embrace necessitism as well. In the main text I always speak of what a spacetime point must be like "if it's a spacetime point", rather than "if it exists", precisely to remain neutral on the issue of necessitism.
} 
possibilities of this sort.

However, once the qualitative metric essentialist eschews SP-necessitism, haecceitistic threats to GR's full determinism quickly recur. We saw in the previous section that the qualitative metric essentialist accommodates the nomic possibility of changing the metric field by claiming that such nomic possibilities differ over which spacetime points there are. These new spacetime points can have essences that differ from the essences of whatever spacetime points there actually are, and so the metric field can change without casting doubt on qualitative metric essentialism. The crucial point to see is that exactly the same move can be used to challenge GR's full determinism. Yes qualitative metric essentialism ensures that we can't get failures of full determinism using nomic possibilities that differ only over the non-qualitative question of which spacetime points play which qualitative roles (because doing so conflicts with points' essences). Still, the doctrine does nothing to prevent us from running an analogous argument using nomic possibilities that differ over the non-qualitative question of which spacetime points there are (and hence also over which spacetime points play which qualitative roles). This problem seems to have been overlooked because those working on the hole argument generally restrict their focus to nomic possibilities that agree about which spacetime points there are when considering potential failures of GR's full determinism, following Earman and Norton's original discussion. Yet it's only in this special case that qualitative metric essentialism helps to preserve GR's full determinism. ${ }^{17}$

So now we run a revised hole argument using two qualitatively identical nomic possibilities that differ over which spacetime points there are only inside some region (the "hole" of this hole argument). The two possibilities agree on which spacetime points there are outside of the hole, and which qualitative roles these points play, so we still get a failure of full determinism: fixing all of the facts up to some time (up to some Cauchy surface to the past of the hole) fails to fix all of the facts throughout spacetime. Whereas Earman and Norton's original argument pointed to indeterminism in which of a fixed class of spacetime points play which qualitative roles inside of the hole, we now have indeterminism in which spacetime points there are playing those qualitative roles inside of the hole. The reason these possibilities can agree on all of the facts outside of the hole (in particular, which spacetime points there are outside of the hole) is because, according to qualitative metric essentialism, spacetime points' essences impose only qualitative constraints on the rest of spacetime, and by hypothesis the two possibilities agree on all qualitative propositions. (Note that I'm addressing the qualitative metric essentialist who doesn't also espouse some

\footnotetext{
${ }^{17}$ This tacit restriction often becomes apparent when authors consider only mathematical solutions of GR that contain the same manifold. If you're then not careful about distinguishing features of your mathematical representation from those of reality itself, you might tacitly take on the substantive assumption that it's nomically necessary which spacetime points there are. I suspect this issue would've been noticed early on had more work on the hole argument discussed modality directly, rather than indirectly via mathematical solution spaces and their representational capacities. To my mind this provides indirect support for my methodological approach from footnote 3 of setting up the issues directly in modal terms, which I'll return to in the conclusion.
} 
non-qualitative essentialist thesis about spacetime that goes beyond her doctrine as defined above.) So qualitative metric essentialists can't argue that the essences of the points outside of the hole somehow preclude there being possibilities of this sort. ${ }^{18}$

What has gone wrong with qualitative metric essentialism? Earman and Norton's hole argument got going because the substantivalist was allegedly forced to admit a certain non-qualitative degree of freedom across nomic possibilities, namely freedom in which points in some fixed class of spacetime points play which qualitative roles. The qualitative metric essentialist purports to block the argument by tying spacetime points to particular qualitative geometric roles conditional on their being spacetime points. We now see that this move doesn't go far enough for those concerned to save GR's full determinism: the doctrine still leaves open a certain non-qualitative degree of freedom across nomic possibilities, namely which spacetime points there are. We saw in section 4 that qualitative metric essentialists must admit some nomic contingency in which spacetime points there are, on pain of accepting SP-necessitism and its Spinozist consequences. However, we now see that they must impose some constraint on how much of this nomic contingency they allow; in particular, if we can swap out which spacetime points there are in some region of spacetime without making some compensatory change at every time outside of that region then we'll get a failure of GR's full determinism, exactly as the original hole argument purported to bring out.

Here's one modal constraint that would do the job:

SP-Clusters: Necessarily, for all spacetime points $o_{1}$ and $o_{2}$, necessarily $o_{1}$ is a spacetime point iff $o_{2}$ is a spacetime point (where both uses of 'necessarily' here express metaphysical necessity).

Picturesquely, SP-clusters ensures that spacetime points come in complete clusters across metaphysical possibilities. The doctrine implies that, for all metaphysical possibilities $w$, and any spacetime point $o$ at $w$, if $o$ were to fail to be a spacetime point then every spacetime point at $w$ would fail to be a spacetime point. Crucially, unlike SP-necessitism, SPclusters allows for variation in which spacetime points there are across nomic possibilities. As a result, qualitative metric essentialists can accept the doctrine without abandoning their diagnosis of the Spinozist threat from section 4.

It's straightforward to see why SP-clusters blocks the revised hole argument I raised for qualitative metric essentialists in this section. The nomic possibilities we needed to run that argument agreed on all of the facts at one time (including which spacetime points

\footnotetext{
${ }^{18}$ The revised hole argument just presented also shows that the "minimal" essentialist conception of spacetime espoused by Healey $(1995,302)$ fails in its ambition to restore GR's full determinism. I won't spell out Healey's proposal here, but it suffices to point out that its modal content amounts to a purely qualitative necessary condition on what spacetime points must be like, and as a result the proposal doesn't differ from qualitative metric essentialism in any respect that's relevant to evaluating whether the proposal addresses my revised hole argument. (The proposal is in fact independent of qualitative metric essentialism, but the reason why stems not from the hole argument but from Healey's desire to also address classical Leibniz shift arguments, which I'm not directly discussing here.)
} 
there are at that time), but differed over which spacetime points there are at some other time. However, SP-clusters ensures that there can't be such nomic possibilities: at all metaphysical possibilities (and so in particular at those where the laws of GR are true), fixing which spacetime points there are at one time fixes which spacetime points there are at all times.

So can qualitative metric essentialists just espouse SP-clusters and rest at ease? The trouble is that qualitative metric essentialism itself doesn't imply SP-clusters: the former imposes only a qualitative modal constraint, whereas the latter imposes a non-qualitative modal constraint. As a result, the qualitative metric essentialist would need to adopt SPclusters as an unexplained modal principle restricting how much contingency there can be in which spacetime points there are. But qualitative metric essentialists should be reluctant to make this move: recall that the main motivation to adopt an essentialist doctrine in the first place was a desire to avoid resting with putatively mysterious modal correlations that cry out for further explanation (like some variety of anti-haecceitism), and instead to explain modal correlations in independent terms. (Note that this point about SP-clusters applies to any modal constraint the qualitative metric essentialist might adopt to block my revised hole argument. For this reason I won't discuss weaker modal constraints that would also do the job.)

Fortunately, there's some good news for those sympathetic to metric essentialism: the revised hole argument I raised in this section affects only qualitative metric essentialism, but can't get going against non-qualitative metric essentialism. Indeed, non-qualitative metric essentialism predicts SP-clusters. This is an attractive result given the metric essentialist's ambition to use essentialist theses to explain the modal constraints we need to bridge the crucial gap between GR's qualitative determinism and its full determinism.

Recall that non-qualitative metric essentialism says that, for every metaphysical possibility $w$, and every spacetime point $o$ at $w$, the complete spacetime description of $w$ according to $o$ is essential to $o$. It's not hard to see why this doctrine implies SP-clusters given the metric essentialist's other commitments (in particular the reduction of modality to essence and the standard assumption that necessarily only true propositions are essential to any objects). First notice that, for every metaphysical possibility $w$, if some spacetime point $o$ at $w$ were to fail to be a spacetime point then the complete spacetime description of $w$ would be false (because it describes which fundamental spatiotemporal properties $o$ instantiates and which fundamental spatiotemporal relations $o$ stands in to other spacetime points). Putting this claim together with non-qualitative metric essentialism, we get the result that, for every metaphysical possibility $w$, if some spacetime point $o_{1}$ at $w$ were to fail to be a spacetime point then every other spacetime point $o_{2}$ at $w$ must also fail to be a spacetime point (because, by non-qualitative metric essentialism, if $o_{2}$ were to still be a spacetime point then the complete spacetime description of $w$ would have to be true, contrary to what we just showed). So we see that non-qualitative metric essentialism implies 
SP-clusters.

Already we can draw a moral from our discussion: those attracted to metric essentialism should prefer non-qualitative metric essentialism to qualitative metric essentialism. Both doctrines block Earman and Norton's hole argument, however non-qualitative metric essentialism also blocks the revised hole argument I raised against qualitative metric essentialism above. Nevertheless, we're about to see that non-qualitative metric essentialism faces problems of its own. ${ }^{19}$

\section{Against Non-Qualitative Metric Essentialism}

In this section I'll raise three worries for non-qualitative metric essentialism. I should flag that the first two worries target only the modal implications of non-qualitative metric essentialism. So these two worries apply equally well to qualitative metric essentialists who adopt SP-clusters as a brute modal constraint, and also to those who espouse only the modal implications of non-qualitative metric essentialism, perhaps due to skepticism about any notion of essence that isn't just shorthand for talk of metaphysical modality.

The first worry is the one I take most seriously. Recall what we wanted from a reply to the hole argument: a modal doctrine which ensures that GR's qualitative determinism implies its full determinism. We assumed that metaphysical possibilities where the laws of GR are true can differ merely haecceitistically only if they differ either over which spacetime points there are or over which spacetime points instantiate which spatiotemporal properties and relations, and thereby limited our attention to haecceitistic threats to GR's full determinism stemming from spacetime. My central worry for non-qualitative metric essentialism is that the doctrine makes it metaphysically impossible for a law to fail to be fully deterministic for either of these reasons. ${ }^{20}$ We wanted some doctrine to ensure that if the laws of GR are qualitatively deterministic then those laws must also be fully deterministic. Non-qualitative metric essentialism secures this result, but in a manner that makes the qualitative determinism of the laws of GR irrelevant to the issue: the laws of GR turn out to be immune to the present threats to full determinism because all laws are!

\footnotetext{
${ }^{19}$ I should flag an important point, implicit in our discussion in this section, that's generally missed: each of the three varieties of anti-haecceitism that we saw in section 2-which are the modal doctrines standardly at issue in discussions of the hole argument-is strictly stronger than what's required for those concerned only to secure GR's full determinism. For example, non-qualitative metric essentialism (equivalently, the combination of qualitative metric essentialism and SP-clusters) allows metaphysical possibilities to differ without differing qualitatively (and indeed allows even metaphysical possibilities at which the laws of GR are true to do so), provided the classes of spacetime points at such possibilities are disjoint. In general, a doctrine can allow for metaphysical possibilities that agree qualitatively yet differ either over which spacetime points there are or over which spacetime points instantiate which spatiotemporal properties and relations, and nonetheless block the relevant threats to full determinism, provided the possibilities differ over at least one proposition at each time. Of course there may be independent considerations that militate against possibilities that differ merely haecceitistically, and hence for adopting some variety of anti-haecceitism.

${ }^{20}$ This was first pointed out to me by Cian Dorr. I've since discovered that distinguished Elvis scholar Gordon Belot has made a related point in print about metric essentialism generally (see footnote 51 of his (2018)).
} 
To see this, note that a threat to GR's full determinism requires nomic possibilities that agree on all of the intrinsic properties of some time to disagree over some proposition. Yet amongst a time's intrinsic properties are those pertaining to which particular spacetime points there are at that time. And given non-qualitative metric essentialism, every spacetime point's essence is so rich that it fixes every other spatiotemporal fact, both qualitative and non-qualitative. As a result, if two nomic possibilities agree on all of the intrinsic properties of some time, they'll agree on all of the spatiotemporal facts, and hence there can't be a haecceitistic threat to GR's full determinism stemming from spacetime. Yet notice that this line of reasoning doesn't rely on any details of the laws of GR; rather it applies to all laws whatsoever given non-qualitative metric essentialism. ${ }^{21}$

Indeed, given either qualitative or non-qualitative metric essentialism, all qualitatively indeterministic laws turn out immune to threats to full determinism stemming from Earman and Norton's hole argument and my revised hole argument. (To see this, note that, given either essentialist doctrine, metaphysical possibilities with qualitatively different spacetimes must contain disjoint classes of spacetime points. As a result, there's no time at which such possibilities agree on all propositions, and hence such possibilities trivially pose no threat to full determinism.) Such laws aren't merely hypothetical: GR itself arguably becomes a qualitatively indeterministic theory if we relax the standard restriction to globally hyperbolic spacetimes (which we made in footnote 4 ) ${ }^{22}$

Remember from section 1 that, according to Earman and Norton, we can't just grant the hole argument's haecceitistic failures of full determinism because the failures are in some sense too easy to produce; as they put it, a metaphysics which automatically gives us failures of full determinism is unacceptable, if full determinism fails "it should fail for a reason of physics" $(1987,524)$ (see also Earman $(1989,180-181)$ ). I raised some doubts about this point; however, the many philosophers working on the hole argument who have

\footnotetext{
${ }^{21}$ You might worry that although properties of a time like containing such-and-such particular spacetime points seem intrinsic, they ought to count as extrinsic once essentialist doctrines like qualitative and non-qualitative metric essentialism are in play. After all, given such doctrines, that a time contains some particular spacetime points necessitates facts about what's going on in the rest of spacetime. Yet the intrinsic qualifiers in my definitions of determinism were precisely there to exclude properties that directly encode information about different times (recall footnote 1). My reply here is that the relevant notion of intrinsicness (and hence of "directly encoding") is hyperintensional. As a result, it can be metaphysically necessary that if a time contains such-and-such particular spacetime points then it's also, say, a time 10 years before a time with so-and-so features, yet the former property can be an intrinsic property of the time despite the latter being one of its extrinsic properties. Here's an analogous case: suppose Kripkean necessity of origins is true, and that I originate from sperm $s$ and egg $e$. Hence, it's metaphysically necessary that if a time instantiates containing Trevor Teitel then it also instantiates being a time later than a time containing s and $e$. Nevertheless, I think the former is an intrinsic property of the present time, whereas the latter is an extrinsic property of the present time. My verdicts about intrinsicness in the case of non-qualitative metric essentialism pattern in the same way. Thanks to Nico Silins for first raising the potential worry here, and for helpful correspondence about the relevant issues.

${ }^{22}$ The reason is that some globally hyperbolic spacetimes admit of multiple non-isometric extensions (for some details see Chrusciel \& Isenberg (1993)). Of course there's a question of whether we can rule out such putative nomic possibilities on independent grounds (e.g. some causality condition), which I won't discuss here. Note that if we move beyond the globally hyperbolic setting we must also modify our assumption that all and only Cauchy surfaces are times at possibilities where GR is true.
} 
found it compelling should also reject metaphysical visions like non-qualitative metric essentialism that automatically vindicate full determinism, with no need for physicists to inquire into what the world's laws are like. We might say to those moved by Earman and Norton's claim: if full determinism holds it should hold for a reason of physics.

We see then that non-qualitative metric essentialism simply doesn't give us the modal implications we sought in an adequate reply to the hole argument: we wanted a doctrine that bridges the gap between some laws being qualitatively deterministic and those laws being immune to the present haecceitistic threats to full determinism, not a doctrine according to which no laws whatsoever can fail to be fully deterministic on these grounds.

Turning to the second and third worries for non-qualitative metric essentialism, these two are suggestive but in my view less compelling than the worry we just saw. The second worry in fact arises for both varieties of metric essentialism. Remember from section 4 that metric essentialists must allow that, holding fixed the laws of GR, which spacetime points there are everywhere in spacetime must change if the value of the stress-energymomentum tensor anywhere in spacetime were to change, where we saw that even tiny qualitative changes would have this result. As a result, the doctrine seems to predict bizarre counterfactuals like 'if I were to have raised my pinky finger yesterday then the total fabric of spacetime would have been different', 'if I were to have snapped my fingers this morning then the region of spacetime actually occupied by Betelgeuse wouldn't have been part of spacetime', and the list could go on.

Despite these predictions, I'm not sure how much weight to put on this second worry. All theories of counterfactuals in deterministic settings have some strange consequences (see Dorr (2016)), plus there are various ways in which metric essentialists can try to explain away our judgments about these bizarre counterfactuals. Still, some may be more moved by the worry from counterfactuals than I am.

Turning to the third worry, notice that non-qualitative metric essentialism implies that the following scenario will be pervasive:

Symmetric Essences: There are distinct objects $o_{1}$ and $o_{2}$ such that (i) some proposition containing $\mathrm{o}_{2}$ is essential to $o_{1}$ and (ii) some proposition containing $o_{1}$ is essential to $o_{2} \cdot{ }^{23}$

So cogent reasons to reject the possibility of symmetric essences would also tell against non-qualitative metric essentialism. Here's the one objection to symmetric essences that I take to have some force, though ultimately I'll suggest that it shouldn't concern nonqualitative metric essentialists.

\footnotetext{
${ }^{23}$ In fact non-qualitative metric essentialism implies the stronger claim that the infinitary analogue of symmetric essences will be pervasive (where the infinitary analogue says that there are infinitely many objects such that, for any of them $o$, some proposition containing all of the others is essential to $o$ ). The issues I discuss for symmetric essences arguably apply even more strikingly to such cases, but I focus on symmetric essences (which requires only two objects) to simply the exposition. Note also that when discussing this third worry from symmetric essences I'll write as if some Russellian theory of propositions is true, and so as if propositions can have objects as constituents, but the worry is compatible with many standard conceptions of propositions.
} 
Some think that a notion of ontological dependence between objects can be reduced to essence. For instance, Fine (1995) proposes that an object $o_{1}$ ontologically depends on another $\mathrm{O}_{2}$ in virtue of there being some proposition containing $o_{2}$ that's essential to $o_{1} \cdot{ }^{24}$ This account seems to get some paradigm cases of ontological dependence right. For instance, the account predicts that singleton-Socrates ontologically depends on Socrates but not vice-versa given the standard judgments about essence stemming from Fine (1994) that we saw above, which some may take as an attractive result.

The worry for symmetric essences is that the essentialist account of ontological dependence predicts that symmetric essences imply symmetric ontological dependence: there are distinct objects $o_{1}$ and $o_{2}$ such that $o_{1}$ ontologically depends on $o_{2}$ yet $o_{2}$ in turn ontologically depends on $o_{1}$. For instance, the account predicts that if non-qualitative metric essentialism is true then, necessarily, for all spacetime points $o, o$ ontologically depends on every spacetime point other than $o$. Yet those theorizing about ontological dependence standardly take the notion to be asymmetric. ${ }^{25}$ Anyone who is sympathetic to the intelligibility of such an asymmetric notion of dependence, and who thinks the notion is reducible to essence, will have reason to reject essentialist doctrines that posit symmetric essences, including non-qualitative metric essentialism.

This worry for symmetric essences generalizes beyond the notion of dependence: if (i) you're sympathetic to the intelligibility of some relation, (ii) you want to reduce that relation to essence in a manner analogous to Fine's account of ontological dependence, and (iii) you have reason to take that relation to be asymmetric, then you'll have reason to reject essentialist doctrines that posit symmetric essences. (Note that the worry also generalizes to those who regard essentialist accounts not as reductions of some allegedly asymmetric relation to essence, but as necessary principles correlating that relation with essence.) I won't survey the candidate relations across contemporary metaphysics that some have taken to satisfy (i), (ii) and (iii), but the list extends beyond ontological dependence (other candidate relations here include "appears in the real definition of", "is more fundamental than", and "partially grounds").

Even though this worry for symmetric essences is quite general, I don't think it need concern non-qualitative metric essentialists, for two reasons. First, the non-qualitative metric essentialist can always argue against the reduction or modal principle tying some allegedly intelligible and asymmetric relation to essence, or instead accept the reduction or

\footnotetext{
${ }^{24} \mathrm{I}^{\prime} \mathrm{m}$ glossing over various details of Fine's account. Most importantly, Fine states the account in terms of his notion of consequential essence with an additional "generalizing out" procedure to avoid various counterexamples. These details aren't important for our purposes; see Fine (1995), Koslicki (2012; 2013), and Tahko \& Lowe (2016) for more details on essentialist accounts of dependence.

${ }^{25}$ For some examples see Schnieder (2006), Cameron (2008), Correia (2008), Schaffer (2009; 2010b) (if it turns out that what goes for Schaffer's entity grounding also goes for dependence), and Tahko \& Lowe (2016). The central arguments I'm aware of aimed directly against the asymmetry of dependence are due to Barnes (2018) and Thompson (2016), also endorsed by Wilson (2014). Of course if we give up asymmetry we must give up either irreflexivity or transitivity, each of which has some precedent (see Jenkins (2011) and Schaffer (2012)), though I'll discuss only asymmetry in the main text.
} 
modal principle yet take her doctrine to establish that we were mistaken to regard that relation as asymmetric. The plausibility of these moves must be taken on a case by case basis, but it's often hard to have strong views one way or the other on the contested abstract fine-grained claims, so the need for such maneuvers will likely be only a small strike against non-qualitative metric essentialism.

Second, non-qualitative metric essentialists with metaphysical commitments that militate against symmetric essences can modify their doctrine so as to keep its modal implications unchanged yet purge it of symmetric essences. For every metaphysical possibility $w$, let $s p_{w}$ be the plurality of all and only the spacetime points at $w \cdot{ }^{26}$ Here's the idea:

Holistic Non-Qualitative Metric Essentialism: For every metaphysical possibility $w$, and every spacetime point $o$ at $w$, the complete spacetime description of $w$ according to $o$ is essential to $s p_{w}$ but not essential to any proper subplurality of $s p_{w}$.

This doctrine agrees with non-qualitative metric essentialism about which propositions are essential to some object or plurality of objects, and hence agrees about which propositions are metaphysically necessary given Fine's reduction (stated in section 3.1). As a result, the doctrine has the same diagnosis of Earman and Norton's hole argument and my revised hole argument as non-qualitative metric essentialism, and is equally vulnerable to the first two worries that we saw in this section. However, holistic non-qualitative metric essentialism isn't committed to symmetric essences, and so is an attractive option for nonqualitative metric essentialists who are moved by the worry from asymmetric relations. I won't speculate here about other pros and cons of moving from non-qualitative metric essentialism to this holistic variant: the modal implications are the same either way, and so I take the first worry that I raised in this section to pose a major problem for both doctrines.

\section{Sufficiency Metric Essentialism}

We saw in section 2 that the most promising avenue in developing a metaphysics of spacetime seemed to be to supplement substantivalism with an essentialist doctrine about spacetime: doing so looked like the best bet to secure the modal constraints stemming from the hole argument in a manner that doesn't cry out for further explanation. But we've seen that extant such doctrines, however understood, simply fail to deliver the right modal implications. What to do?

The problems for qualitative and non-qualitative metric essentialism that we've seen suggest the way forward in our search for an adequate essentialist doctrine about spacetime. First, we need a doctrine that, unlike qualitative metric essentialism, in fact bridges

\footnotetext{
${ }^{26} \mathrm{My}$ talk of the plurality of such-and-such objects throughout should not be understood as requiring there to be some further object in addition to whatever objects make up the plurality (unlike, say, the set or mereological fusion of those objects, as standardly conceived).
} 
the crucial gap between GR's qualitative determinism and its full determinism (thereby resolving both the original hole argument and my revised hole argument). Yet, unlike non-qualitative metric essentialism, the doctrine mustn't render the relevant haecceitistic failures of full determinism metaphysically impossible. Also, some may be more moved by the second and third worries from the last section than I was, so ideally the essentialist doctrine will avoid the commitments that lead to the worry from bizarre counterfactuals, and also won't posit symmetric essences. I'll now propose an essentialist metaphysics of spacetime with exactly these features. Unfortunately, we'll ultimately see that nevertheless essentialist doctrines are in trouble, and hence we have to look elsewhere for an adequate reply to the hole argument.

Notice that so far we've looked at essentialist doctrines that impose only necessary conditions on spacetime points: doctrines which imply that, for every metaphysical possibility $w$ and every spacetime point $o$ at $w$, if $o$ is a spacetime point then it must have such-andsuch properties. Essentialist doctrines about any subject matter generally take this form of implying only necessary conditions, and it's the form most naturally suggested by the metric essentialist's slogan that spacetime points stand in their metric relations essentially. The essentialist doctrine I want to propose takes a different form, with modal implications that aren't necessary conditions on what objects must be like if they're to be spacetime points, but rather sufficient conditions for objects to play certain qualitative geometric roles and thereby be spacetime points.

Before stating the doctrine, we need some new notation and definitions. Starting with the new notation, I'll use oo as a plural variable ranging over both individual spacetime points and pluralities of multiple spacetime points (and recall footnote 26 for more on this 'plurality' talk). Turning to the new definitions, first, for any metaphysical possibility $w$, we define $w^{\prime}$ s complete qualitative spacetime description as the proposition that details the complete qualitative pattern of instantiation at $w$ of the fundamental spatiotemporal properties and relations, and nothing else. Next, for any metaphysical possibility $w$, and any individual spacetime point or plurality of spacetime points oo at $w$, we define $w$ 's unconditional complete qualitative spacetime description according to oo as the proposition that the complete qualitative pattern of instantiation at $w$ of the fundamental spatiotemporal properties and relations obtains and oo plays the qualitative geometric role in that structure that it in fact plays at $w$. Given a sentence expressing the complete spacetime description of some possibility (defined in section 3.2), if we existentially generalize on every occurrence of a name for a spacetime point then the resulting quantified sentence expresses the complete qualitative spacetime description of that possibility, whereas if we existentially generalize on every occurrence of a name for a spacetime point besides the names for the point or points in oo then the resulting quantified sentence expresses the unconditional complete qualitative spacetime description of that possibility according to $o o$.

Almost there. We now define, for any metaphysical possibility $w$, and any individual 
spacetime point or plurality of spacetime points $o o$ at $w, o o$ 's conditional geometric role proposition at $w$ : the proposition that if $w^{\prime}$ s complete qualitative spacetime description obtains then $w$ 's unconditional complete qualitative spacetime description according to oo obtains. Roughly, $o o^{\prime}$ s conditional geometric role proposition at a metaphysical possibility $w$ says that if the complete qualitative geometric structure at $w$ obtains then oo plays the qualitative geometric role in that structure that it in fact plays at $w$. With this definition in hand, we can state the following essentialist doctrine:

Sufficiency Metric Essentialism: For every metaphysical possibility $w$, and every individual spacetime point or plurality of spacetime points $o o$ at $w, o o$ 's conditional geometric role proposition at $w$ is essential to oo.

The implications of this essentialist doctrine for metaphysical modality, given Fine's reduction, will be familiar: sufficiency metric essentialism implies SP-anti-haecceitism, which we know suffices to block the haecceitistic threats to GR's full determinism stemming from both Earman and Norton's hole argument and my revised hole argument, and thereby bridge the crucial gap between GR's qualitative and full determinism given our focus here. According to sufficiency metric essentialism, once you fix the qualitative geometric facts you fix which spacetime points there are and which spatiotemporal properties and relations those points instantiate. ${ }^{27}$ More precisely, the doctrine predicts that no metaphysical possibilities differ either over which spacetime points there are or over which spacetime points instantiate which spatiotemporal properties and relations without also differing over the qualitative structure of spacetime, which is just SP-anti-haecceitism. ${ }^{28}$

Because sufficiency metric essentialism blocks my revised hole argument, the doctrine succeeds where qualitative metric essentialism failed. The doctrine also avoids all three worries that we saw for non-qualitative metric essentialism. First, notice that a spacetime

\footnotetext{
${ }^{27}$ Officially sufficiency metric essentialism fixes only which spacetime points there are and which fundamental spatiotemporal properties and relations those points instantiate. However, the more general claims in the main text follow provided the pattern of instantiation of spatiotemporal properties and relations supervenes on the pattern of instantiation of fundamental spatiotemporal properties and relations. I've taken this plausible claim for granted at various points above, and will continue to adopt it throughout.

${ }^{28}$ We need to formulate sufficiency metric essentialism in terms of both individual spacetime points and pluralities of spacetime points because of the nomic possibility of general relativistic spacetimes with qualitative symmetries, i.e. those where some qualitative geometric role is played by more than one spacetime point. By permuting some spacetime points that play the multiply realized qualitative geometric role at such possibilities, we can generate merely haecceitistic threats to GR's full determinism all the while preserving which spacetime points there are and which individual spacetime points play which qualitative geometric roles. However, once we take into account pluralities of spacetime points we block this construction. (Why? Well to threaten full determinism we must leave the possibilities unchanged at some time $t$. As a result, when we permute some spacetime points that play the multiply realized qualitative geometric role, we'll inevitably change the pattern of instantiation of spatiotemporal properties and relations by some plurality of spacetime points that includes at least one of the permuted spacetime points as well as at least one spacetime point at t.) To put the point another way, sufficiency metric essentialism implies SP-anti-haecceitism (which we know suffices to bridge GR's qualitative and full determinism) only because the doctrine encompasses pluralities of spacetime points in addition to individual spacetime points. Thanks to an anonymous referee for making clear to me the important issues raised by symmetric spacetimes, which occasioned significant improvements throughout the paper.
} 
point or plurality of spacetime points $o o^{\prime}$ s conditional geometric role proposition at any possibility doesn't contain any objects other than those in $o o$, and so sufficiency metric essentialism isn't committed to symmetric essences. Second, note that sufficiency metric essentialism itself is neutral on the truth of any essentialist or modal thesis that takes the traditional form of implying a necessary condition on what an object must be like if it's to be spacetime point. So the doctrine is compatible with visions where the very same object can be a spacetime point at possibilities that ascribe radically different complete qualitative structures to spacetime. As a result, unlike extant essentialist visions of spacetime, sufficiency metric essentialism doesn't predict the problematic counterfactuals that we saw in section 6, and isn't even vulnerable to the Spinozist threat from section 4 (indeed sufficiency metric essentialists can even adopt SP-necessitism should they be so inclined). Finally, and most importantly, sufficiency metric essentialism allows that possibilities differing over the complete qualitative structure of spacetime can nevertheless agree on all of the intrinsic properties of a time, and hence the doctrine allows that laws which fail to be qualitatively deterministic can also fall foul of the haecceitistic threats to full determinism brought out by Earman and Norton's hole argument and my revised hole argument. As a result, the doctrine secures GR's full determinism only given GR's qualitative determinism, rather than by making any possible laws whatsoever immune to the relevant haecceitistic threats to full determinism. For this reason sufficiency metric essentialism avoids the central problem that we saw for non-qualitative metric essentialism, and instead actually gives us the modal implications we sought in an adequate reply to the hole argument.

\section{Conclusion: A Return to Modality}

We've seen that sufficiency metric essentialism is where those sympathetic to bridging the gap between GR's qualitative determinism and its full determinism with an essentialist doctrine should place their allegiances. Indeed the doctrine avoids every worry we saw for both qualitative metric essentialism and non-qualitative metric essentialism. Metric essentialist slogans have always been standard members in surveys of replies to the hole argument, and now we know what an essentialist doctrine about spacetime should look like if it's to in fact address the argument. Moreover, we saw in section 2 that the common desire to further explain modal replies to the hole argument like SP-anti-haecceitism provides ample motivation for adopting an essentialist doctrine about spacetime. Additional motivation came from the challenges we saw for the main alternative option for doing so, namely spacetime structuralism. Putting all of this together, I'm sometimes tempted to just stop here, and to regard our results so far as an extended argument for a new essentialist metaphysics of spacetime, namely sufficiency metric essentialism.

I suspect that this conclusion may even be attractive to some spacetime structuralists. 
Recall from section 2 that it's an open question whether there's a precise and intelligible conception of spacetime in the vicinity of structuralist slogans that doesn't collapse into some other reply. I want to offer sufficiency metric essentialism as a doctrine structuralists about spacetime should take a serious look at when searching for a precise metaphysics of spacetime to make good on their various slogans. The resulting vision doesn't make good on all structuralist claims (e.g. we still have objects around), but it certainly has the flavor of some things structuralists say (slogans like that objects have their 'identities explained in terms of places in structures', plus the doctrine implies SP-anti-haecceitism given Fine's reduction). Most importantly, sufficiency metric essentialism at least offers a precise doctrine we can evaluate. ${ }^{29}$

So what's the problem? Despite everything I just said, I want to suggest a different conclusion to draw from our discussion. Now that we know what an essentialist doctrine about spacetime must look like in order to address the hole argument, I think the conclusion to draw is that no such doctrine can do the work that motivates them in the first place. The reason is that essentialist doctrines about spacetime can't fulfill their dual ambition of implying the right modal constraints to address the hole argument while at the same time dispelling the mystery as to why such modal constraints obtain. We've seen that in order to deliver the right modal constraints an essentialist doctrine must take a sufficiency form, like sufficiency metric essentialism. But I'll soon argue that as a result the doctrine becomes unexplanatory. This conclusion will give us cause to reconsider the anti-modality assumptions that motivated essentialist doctrines in the first place, and lend support to the familiar reply to the hole argument of simply supplementing substantivalism with the requisite modal constraints, e.g. some variety of anti-haecceitism, but not insisting on further explaining why these constraints obtain. I'll now motivate this alternative conclusion, and along the way draw some morals for contemporary metaphysics as well as future work on spacetime and the metaphysics of physics more generally.

\subsection{Essentialism and Explanation}

Recall that the supposed problem with responding to the hole argument by just laying down SP-anti-haecceitism itself was that this modal constraint seemed mysterious, and to cry out for further explanation. Additional support for this worry came from the trend in metaphysics of claiming that it's problematic for explanation to stop with modal theses, and that instead we should try to explain why modal theses obtain in terms of underlying hyperintensional doctrines, most prominently in terms of underlying essentialist doctrines.

Now try to put yourself in the mindset of someone who claims on these grounds

\footnotetext{
${ }^{29} \mathrm{We}^{\prime}$ ve been discussing spacetime, but I should add that structuralists about other domains (such as mathematics) could appeal to an essentialist thesis analogous to sufficiency metric essentialism in order to make good on their claims, though the plausibility of the doctrine must be taken on a case by case basis.
} 
that an adequate reply to the hole argument must go further than some variety of antihaecceitism: you can't just lay down a modal constraint like SP-anti-haecceitism, they'll claim, but rather you must explain why it obtains in independent terms. My worry is that I don't think anyone in this mindset should take their worries to be allayed by sufficiency metric essentialism. The doctrine simply works backwards from SP-anti-haecceitism, and shoehorns that modal constraint into essentialist terms: sufficiency metric essentialism decomposes the general modal constraint of SP-anti-haecceitism into individual constraints (conditional geometric role propositions) on each spacetime point or plurality of spacetime points $o o$, and then says every $o o^{\prime}$ s individual constraint is essential to it. Yet to the extent that there's anything mysterious about SP-anti-haecceitism itself, the mystery hasn't gone away: only now we'll wonder about why all of those propositions are essential to all of those spacetime points! The worry isn't just that it's mysterious, for some particular oo, why $o o^{\prime}$ s conditional geometric role proposition is essential to oo (here the sufficiency metric essentialist might reply with speeches like 'that's just what it is to be oo!'). The more striking mystery that's harder to shake off is why there are only spacetime points with these particular rich essential profiles rather than some other less rich essential profiles. In general, out of all the possible ways spacetime points' essences could've turned out, why are they fine-tuned in just the way required to vindicate sufficiency metric essentialism? This strong essentialist constraint doesn't seem any less mysterious than the modal constraint of SP-anti-haecceitism itself, even though the modal constraint is strictly speaking implied by the essentialist one if we assume Fine's reduction of modality to essence. So sufficiency metric essentialism doesn't fulfill the desire that motivated going beyond the standard modal reply to the hole argument and seeking some essentialist reply in the first place. $^{30}$

Notice, then, that here we have a case where even if we concede that a modal thesis obtains in virtue of an underlying hyperintensional one, we don't seem to gain the right kind of explanatory advance over the modal thesis itself, because any supposed air of mystery in the modal correlation recurs. ${ }^{31}$ But didn't Fine's (1994) case of Socrates and singletonSocrates (summarized in section 3.1) teach us that essentialist theses satisfy our thirst to further explain why modal ones obtain? I think there's an important rift in our essentialist judgments here. In the core cases that motivate Fine's hyperintensional notion of essence, and his reduction of modality to essence as well, we have a judgment that the essentialist

\footnotetext{
${ }^{30}$ Sufficiency metric essentialism is also vulnerable to the worry from footnote 8 about arbitrariness in which particular qualitative roles spacetime points can play across metaphysical possibilities. One straightforward response to this worry would be to supplement the doctrine with non-qualitative metric essentialism. But we saw the problems with this doctrine above.

${ }^{31}$ Dasgupta (2011) argues that a problem with standard anti-haecceitist views is that they make what he calls a "bare modal claim", by which he means that their modal doctrine follows "not from [their] views about the metaphysics of the manifold, but rather from [their] independent views about modality in general" (140-141). In this terminology, and assuming sufficiency metric essentialism counts as a view about the "metaphysics of the manifold", the moral in the main text is that sometimes non-bare modal claims are no less mysterious than bare modal claims.
} 
claims successfully dispel our sense that the modal claims are mysterious and cry out for further explanation: for example, starting with the essentialist judgment that singletonSocrates essentially contains Socrates (that's 'what it is to be singleton-Socrates'), seems to genuinely satisfy our desire to further explain why it's necessary that the singleton contains Socrates if both exist. The trouble is that once we move beyond the hospitable and circumscribed range of modal claims that motivate the program of reducing modality to essence, showing a modal claim to obtain in virtue of an essentialist one still leaves us with the phenomenology of mysteriousness that we started out with.

One salient contrast comes when the modal proposition at issue doesn't take the traditional form of imposing necessary conditions on what objects must be like if they exist, such as necessitated propositions that instead take a sufficiency form (e.g. necessitated conditional geometric role propositions).$^{32}$ If that's right then we have a general dilemma for essentialist doctrines about spacetime, given their dual ambitions stated above: in order to deliver the right modal implications to bridge GR's qualitative and full determinism, essentialist doctrines must take a sufficiency form, but then they'll be just as mysterious as the modal implications themselves. Sufficiency metric essentialism might then be unexplanatory precisely because it takes a sufficiency form, as it must to deliver the requisite modal constraints.

This result doesn't straightforwardly deliver the stronger moral that there's something problematic about the program of reducing modality to essence itself. After all, Fine's reduction is an 'in virtue of' claim (often called a 'grounding claim' or a 'metaphysical explanation'). And 'in virtue of' claims needn't in general correlate with the sense of explanation we take to be relevant to dispelling our sense that some claim is mysterious and cries out for further explanation (call the latter the 'mystery-dissolving' sense of explanation). Nevertheless, I suspect that what motivates some to adopt Fine's reduction is precisely the thought that modal constraints are often mysterious and cry out for further explanation, and that showing how they obtain in virtue of essentialist theses will generally help to make mysteries of this sort go away (notice that this was exactly the hope with SP-anti-haecceitism and essentialist replies to the hole argument). For proponents of the view with these motivations, my results constitute good reason to look elsewhere for a reduction of metaphysical modality, or else to accept that some facts about metaphysical modality themselves are fundamental. ${ }^{33}$ This upshot casts doubt on aspects of the recent trend against modality in metaphysics, with its desire to show that modal facts obtain in virtue of underlying hyperintensional ones. However, even the weaker moral above goes against aspects of current wisdom: we've seen that even granting that modal facts obtain

\footnotetext{
${ }^{32}$ Other cases where essentialist doctrines seem unhelpful concern those that would be needed to ground reasonably complex modal propositions, such as propositions involving iterated modalities. I discuss this point in my (2019b).

${ }^{33}$ This moral is of a piece with conclusions I defended in my (2019b), where I raised independent worries for the program of reducing modality to essence.
} 
in virtue of underlying hyperintensional ones, the order of methodological priority can run against the order of metaphysical priority. If our only grip on the underlying hyperintensional facts requires us to work backwards from which modal constraints we want to vindicate (as seemed to be the case with sufficiency metric essentialism), then we should still be theorizing directly in modal terms, even if we don't adopt the stronger conclusion that places modality first also in the order of metaphysical priority.

\subsection{The Way Forward}

I'll now sketch my views on the way forward in light of all this for work on the metaphysics of spacetime and the hole argument. I'll then close with a moral for the metaphysics of physics generally.

First, our discussion gives us cause to reconsider the familiar reply to the argument that we saw in section 2: simply lay down some variety of anti-haecceitism to supplement substantivalism, and say nothing further. We saw that such a modal constraint strikes us as mysterious, and to cry out for further explanation. However, now we know that even the best essentialist reply will leave us with the same thirst for further explanation, which undercuts the main motivation for going beyond a modal reply and seeking an essentialist reply in the first place. The lesson I draw is that we just need to get used to accepting some seemingly mysterious facts somewhere in our conception of reality, and that perhaps we should conclude that explanation in the mystery-dissolving sense can simply end with independently motivated modal doctrines like SP-anti-haecceitism.

But what about other options for grounding modal constraints like SP-anti-haecceitism in independent terms? Perhaps one of these will successfully dispel our sense that it's mysterious why SP-anti-haecceitism obtains? The trouble is that every alternative option is either an independently problematic object-free view, or else faces an analogue of my arguments against essentialist replies. Starting with the latter horn, the family of views I have in mind are those that try to make SP-anti-haecceitism seem less mysterious by appealing to hyperintensional resources yet while still countenancing objects (and in particular spacetime points). On any such attempt we'll need to impose some hyperintensional constraints on all spacetime points which purport to limit their modal freedom (and hence imply SP-anti-haecceitism). If the constraint takes the form of a necessary condition, it will be either qualitative or non-qualitative. If qualitative, the view will face my revised hole argument, if non-qualitative the view will face the first objection I raised against nonqualitative metric essentialism above (from making determinism too cheap). Yet if the constraint takes the sufficiency form needed to imply SP-anti-haecceitism, then we'll be left wondering about why there are spacetime points with this particular profile of rich hyperintensional constraints rather than some other one. Thus my arguments taken together suggest that there's no middle ground between the extremes of my preferred brute antihaecceitist conclusion and the "qualitativist" structuralist view that I sketched in section 
2, which purports to do without spacetime points: the latter seems like the only potential option that might rid us of mysteries akin to why SP-anti-haecceitism should be true. Unfortunately, we saw that these object-free conceptions of reality face problems, and are very much works in progress. Thus, although our discussion provides impetus to keep investigating the prospects of views that do without spacetime points, at this stage of inquiry the brute anti-haecceitist position looks to me like the best bet.

Note that the brute anti-haecceitist position isn't that we should countenance any modal constraints whatsoever, however mysterious they seem, but rather only those with some independent motivation. An analogy that may help you come around to the view is the debate over conditional excluded middle, the principle that for all propositions $p$ and $q$, either $p$ counterfactually implies $q$ or $p$ counterfactually implies $\neg q$. Many accept the principle on general theoretical grounds, despite it often committing them to mysterious modal facts about the closeness of possibilities that seem to cry out for further explanation. Channeling this attitude, proponents of the standard anti-haecceitist reply can say that their modal doctrine is motivated on general theoretical grounds-preserving GR's full determinism without rendering it metaphysically impossible for there to be laws that are vulnerable to the relevant haecceitistic threats to full determinism-despite it committing them to mysterious seemingly brute modal facts. ${ }^{34}$

Finally, let me draw a moral for the metaphysics of physics more broadly. ${ }^{35}$ As I mentioned in footnote 3, I deliberately set up the issues surrounding the hole argument by discussing modality directly, and which doctrines imply the right modal correlations to bridge GR's qualitative and full determinism, rather than instead following the standard practice in the literature of theorizing primarily in terms of mathematical solution spaces and what we use them to represent. One example that illustrates the fruits of this methodological shift was my revised hole argument: this argument pointed to an issue that had arguably been hidden from view by the standard focus on the mathematical formalism of GR to the exclusion of directly discussing the phenomena we use this formalism to represent, in this case nomic modality (see footnote 17). This methodological shift also shows that certain extant replies to the hole argument can't possibly aspire to fully address the issues that the argument brings out. I have in mind purely syntactic approaches that focus only on the formalism of GR (see for example Mundy (1992) and Leeds (1995)), and also approaches that focus primarily on the representational conventions practicing physicists adopt when using the formalism of GR to describe the non-mathematical world (see especially Weatherall (2018)). Again, we can state a version of the hole argument without ever mentioning mathematical solution spaces and their representational capacities. So

\footnotetext{
${ }^{34}$ Proponents of conditional excluded middle often appeal to vagueness to mitigate the mysteriousness of their doctrine (see, for example, Stalnaker (1981), Cross (2009), and Bacon (2015)). Anti-haecceitists can adopt the same strategy to address some complaints about their modal doctrine; in particular, pleading vagueness seems like a promising strategy to address the worry I raised in footnote 8 .

${ }^{35}$ For a similar moral see the conclusion of my (2019a).
} 
absent independent arguments for taking my purely modal statement of the problem to be somehow illegitimate, these approaches at best address only some issues in the vicinity of extant discussions of the hole argument. In general, then, I take our discussion to illustrate the fruitfulness of sometimes approaching topics in the philosophy of physics in a more metaphysics-oriented spirit, where we don't shy away from directly discussing whatever phenomenon is at issue. We've seen throughout how bringing to bear work from contemporary metaphysics (especially the metaphysics of modality in this particular case) can help us move the conversation forward on a central topic in the philosophy of physics. We've also seen how the influence can run in the other direction, by showing how reflecting on a central topic in the foundations of physics (the hole argument) can inform some central questions in contemporary metaphysics. So let me close with a plea for both metaphysicians and philosophers of physics to keep an open mind about what they can learn from each other. ${ }^{36}$

\footnotetext{
${ }^{36}$ Thanks most of all to Cian Dorr, for extremely helpful comments and advice at every stage of the paper's development. I'm also deeply indebted to David Albert, Gordon Belot, Ben Holguín, Tim Maudlin, and the anonymous reviewers, for extensive comments and illuminating discussion. Finally, for helpful comments and discussion about this material, many thanks to Andreas Ditter, Hartry Field, Jane Friedman, Jake Nebel, Nico Silins, Daniel Viehoff, David Wallace, and audiences at Cornell University, New York University, and the University of Toronto.
} 


\section{References}

Arntzenius, Frank. 2012. Space, Time, and Stuff. Oxford: Oxford University Press.

Bacon, Andrew. 2015. Stalnaker's Thesis in Context. The Review of Symbolic Logic 8(1). 131-163.

Barnes, Elizabeth. 2018. Symmetric Dependence. In Ricki Bliss \& Graham Priest (eds.), Reality and its Structure: Essays in Fundamentality, 50-69. Oxford: Oxford University Press.

Belot, Gordon. 1995. New work for counterpart theorists: Determinism. The British journal for the philosophy of science 46(2). 185-195.

Belot, Gordon. 2018. Fifty Million Elvis Fans Can't be Wrong. Nous 52(4). 946-981.

Brighouse, Carolyn. 1994. Spacetime and Holes. PSA: Proceedings of the Biennial Meeting of the Philosophy of Science Association 1. 117-125.

Brighouse, Carolyn. 1997. Determinism and Modality. The British Journal for the Philosophy of Science 48(4). $465-481$.

Cameron, Ross P. 2008. Turtles all the Way Down: Regress, Priority and Fundamentality. The Philosophical Quarterly 58(230). 1-14.

Chrusciel, Piotr T. \& James Isenberg. 1993. Nonisometric vacuum extensions of vacuum maximal globally hyperbolic spacetimes. Physical Review D 48(4). 1616-1628.

Correia, Fabrice. 2008. Ontological Dependence. Philosophy Compass 3(5). 1013-1032.

Cross, Charles B. 2009. Conditional Excluded Middle. Erkenntnis (1975-) 70(2). 173-188.

Dasgupta, Shamik. 2009. Individuals: an essay in revisionary metaphysics. Philosophical Studies 145(1). 35-67.

Dasgupta, Shamik. 2011. The Bare Necessities. Philosophical Perspectives 25(1). 115-160.

Dasgupta, Shamik. 2017. Can We Do Without Fundamental Individuals? Yes. In Elizabeth Barnes (ed.), Current Controversies in Metaphysics, 7-23. New York: Routledge.

Dorr, Cian. 2010. Review of Every Thing Must Go: Metaphysics Naturalized. Notre Dame Philosophical Reviews .

Dorr, Cian. 2016. Against Counterfactual Miracles. Philosophical Review 125(2). 241-286.

Earman, John. 1989. World Enough and Space-Time. Cambridge: MIT Press.

Earman, John \& John Norton. 1987. What price spacetime substantivalism? The hole story. British Journal for the Philosophy of Science 38. 515-525.

Field, Hartry. 1984. Can We Dispense with Space-Time? PSA:Proceedings of the Biennial Meeting of the Philosophy of Science Association 2. 33-90. Citations to reprinted version in Field 1989, Realism, Mathematics, and Modality, 171-226. Oxford: Basil Blackwell.

Fine, Kit. 1994. Essence and Modality. Philosophical Perspectives 8. 1-16.

Fine, Kit. 1995. Ontological Dependence. Proceedings of the Aristotelian Society 95. 269-290.

Greaves, Hilary. 2011. In Search of (spacetime) Structuralism. Philosophical Perspectives 25(1). 189-204.

Hawthorne, John. 2006. Determinism De Re. In Metaphysical Essays, 239-243. Oxford: Oxford University Press.

Healey, Richard. 1995. Substance, Modality and Spacetime. Erkenntnis (1975-) 42(3). 287-316.

Hoefer, Carl. 1996. The Metaphysics of Space-Time Substantivalism. The Journal of Philosophy 93(1). 5-27.

Jenkins, C. S. 2011. Is Metaphysical Dependence Irreflexive? The Monist 94(2). 267-276. 
Koslicki, Kathrin. 2012. Varieties of Ontological Dependence. In Fabrice Correia \& Benjamin Schnieder (eds.), Metaphysical Grounding: Understanding the Structure of Reality, 186-213. Cambridge University Press.

Koslicki, Kathrin. 2013. Ontological Dependence: An Opinionated Survey. In Benjamin Schnieder, Miguel Hoeltje \& Alex Steinberg (eds.), Varieties of Dependence: Ontological Dependence, Grounding, Supervenience, Response-Dependence (Basic Philosophical Concepts), 31-64. Philosophia Verlag.

Ladyman, James. 2014. Structural Realism. In Edward N. Zalta (ed.), The Stanford Encyclopedia of Philosophy, .

Ladyman, James \& Don Ross. 2007. Every Thing Must Go: Metaphysics Naturalized. Oxford: Oxford University Press.

Lam, Vincent \& Michael Esfeld. 2012. The Structural Metaphysics of Quantum Theory and General Relativity. Journal for General Philosophy of Science 43(2). 243-258.

Leeds, Stephen. 1995. Holes and Determinism: Another Look. Philosophy of Science 62(3). 425-437.

Lowe, E. J. 2012. Asymmetrical Dependence in Individuation. In Fabrice Correia \& Benjamin Schnieder (eds.), Metaphysical Grounding: Understanding the Structure of Reality, 214-233. Cambridge University Press.

Maudlin, Tim. 1988. The Essence of Space-Time. PSA: Proceedings of the Biennial Meeting of the Philosophy of Science Association 1988. 82-91.

Maudlin, Tim. 1990. Substances and space-time: What Aristotle would have said to Einstein. Studies in History and Philosophy of Science Part A 21(4). 531-561.

Maudlin, Tim. 2007. A Modest Proposal Concerning Laws, Counterfactals, and Explanations. In The Metaphysics Within Physics, 5-49. Oxford: Oxford University Press.

Melia, Joseph. 1999. Holes, Haecceitism and Two Conceptions of Determinism. The British Journal for the Philosophy of Science 50(4). 639-664.

Mundy, Brent. 1992. Space-Time and Isomorphism. PSA: Proceedings of the Biennial Meeting of the Philosophy of Science Association 1992. 515-527.

Norton, John D. 2015. The Hole Argument. In Edward N. Zalta (ed.), The Stanford Encyclopedia of Philosophy, Metaphysics Research Lab, Stanford University fall 2015 edn.

Pooley, Oliver. 2006. Points, Particles and Structural Realism. In Dean Rickles, Steven French \& Juha Saatsi (eds.), The Structural Foundations of Quantum Gravity, 83-120. Oxford University Press.

Pooley, Oliver. 2013. Substantivalist and Relationalist Approaches to Spacetime. In Robert Batterman (ed.), The Oxford Handbook of Philosophy of Physics, 522-586. Oxford: Oxford University Press.

Russell, Jeffrey Sanford. 2016. Qualitative Grounds. Philosophical Perspectives 30(1). 309-348.

Schaffer, Jonathan. 2009. On What Grounds What. In David Manley, David J. Chalmers \& Ryan Wasserman (eds.), Metametaphysics: New Essays on the Foundations of Ontology, 347-383. Oxford University Press.

Schaffer, Jonathan. 2010a. The Internal Relatedness of All Things. Mind 119(474). 341-376.

Schaffer, Jonathan. 2010b. Monism: The Priority of the Whole. Philosophical Review 119(1). 31-76.

Schaffer, Jonathan. 2012. Grounding, Transitivity, and Contrastivity. In Fabrice Correia \& Benjamin Schnieder (eds.), Metaphysical Grounding: Understanding the Structure of Reality, 122-138. Cambridge University Press.

Schnieder, Benjamin Sebastian. 2006. A Certain Kind of Trinity: Dependence, Substance, Explanation. Philosophical Studies 129(2). 393-419.

Sider, Theodore. 2011. Writing the Book of the World. Oxford: Oxford University Press.

Stalnaker, Robert C. 1981. A Defense of Conditional Excluded Middle. In William L. Harper, Robert Stalnaker \& Glenn Pearce (eds.), Ifs: Conditionals, Belief, Decision, Chance and Time The University of Western Ontario Series in Philosophy of Science, 87-104. Dordrecht: Springer Netherlands. 
Tahko, Tuomas E. \& E. J. Lowe. 2016. Ontological Dependence. In Edward N. Zalta (ed.), The Stanford Encyclopedia of Philosophy, .

Teitel, Trevor. 2019a. Background independence: Lessons for further decades of dispute. Studies in History and Philosophy of Modern Physics 65. 41-54.

Teitel, Trevor. 2019b. Contingent Existence and the Reduction of Modality to Essence. Mind 128(509). 39-68.

Thompson, Naomi. 2016. Metaphysical Interdependence. In Mark Jago (ed.), Reality Making, 38-56. Oxford: Oxford University Press.

Turner, Jason. 2017. Can We Do Without Fundamental Individuals? No. In Elizabeth Barnes (ed.), Current Controversies in Metaphysics, 24-34. New York: Routledge.

Van Cleve, James. 1985. Three Versions of the Bundle Theory. Philosophical Studies 47(1). 95-107.

Weatherall, James Owen. 2018. Regarding the 'Hole Argument'. The British Journal for the Philosophy of Science 69(2). 329-350.

Williamson, Timothy. 2013. Modal Logic as Metaphysics. Oxford: Oxford University Press.

Williamson, Timothy. 2016. Modal science. Canadian Journal of Philosophy 46(4-5). 453-492.

Wilson, Jessica M. 2014. No Work for a Theory of Grounding. Inquiry 57(5-6). 535-579. 